\title{
Intermittency, current flows, and short time diffusion in interacting finite sized one-dimensional fluids
}

\author{
Subrata Pal, Goundla Srinivas, Sarika Bhattacharyya, and Biman Bagchia),b) \\ Solid State and Structural Chemistry Unit, Indian Institute of Science, Bangalore 560 012, India
}

\begin{abstract}
Long time molecular dynamics simulations of one-dimensional Lennard-Jones systems reveal that while the diffusion coefficient of a tagged particle indeed goes to zero in the very long time, the mean-square displacement is linear with time at short to intermediate times, allowing the definition of a short time diffusion coefficient [Lebowitz and Percus, Phys. Rev. 155, 122 (1967)]. The particle trajectories show intermittent displacements, surprisingly similar to the recent experimental results [Wei et al., Science 287, 625 (2000)]. A self-consistent mode coupling theory is presented which can partly explain the rich dynamical behavior of the velocity correlation function and also of the frequency dependent friction. The simulations show a strong dependence of the velocity correlation function on the size of the system, quite unique to one dimensional interacting systems. Inclusion of background noise leads to a dramatic change in the profile of the velocity time correlation function, in agreement with the predictions of Percus [Phys. Rev. A 9, 557 (1974)].
\end{abstract}

\section{INTRODUCTION}

Diffusion in one-dimensional (1D) fluids, sometimes also referred to as "single file systems," ${ }^{1}$ is a subject of great current interest, not only from practical importance as transport in zeolites and nanotubes, ${ }^{2-5}$ but also as a problem of great, long standing fundamental importance. ${ }^{6-12}$ The first theoretical study was carried out by Jepsen ${ }^{6}$ who presented an analytical solution for the velocity correlation function of hard rods. Lebowitz and Percus ${ }^{11}$ studied a short time limit of this function and derived expressions for short time diffusion coefficient. Note that in the limit of infinite system the transmission rate of a signal across the system is also infinity and thus this short time diffusion should persist for an infinitely long time. Bishop et al. ${ }^{13}$ calculated the diffusion coefficient in 1D (D refers to the dimensionality) LennardJones fluids. Earlier we have presented a simulation and mode coupling theory analysis of this problem and in particular investigated the origin of the $t^{-3}$ time $(t)$ dependence of the velocity time correlation function in the long time. Recently, several studies have been devoted to diffusion in zeolite channels and nanotubes. ${ }^{2,4}$ Here one often studies diffusion in low fluid concentration but there is a background noise term which can alter the diffusion behavior. Very recently, diffusion has been studied in the limit of high loading (meaning high fluid concentration), which makes the two different schools on one-dimensional diffusion to converge.

Diffusion coefficient $(D)$ in 1D can be defined by any of the following two definitions,

$$
D=\lim _{t \rightarrow \infty} \frac{1}{2 t}\left\langle\Delta x^{2}(t)\right\rangle
$$

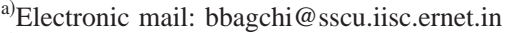

b) Also at the Jawaharlal Nehru Center for Advanced Scientific Research, Jakkur, Bangalore.
}

and

$$
D=\int_{0}^{\infty} d t\langle v(0) v(t)\rangle
$$

where $\left\langle\Delta x^{2}(t)\right\rangle$ is the mean-square displacement (MSD) of a tagged particle, and $v(t)$ is the velocity at time $t$.

The self-diffusion coefficient in liquids shows strong dependence on dimensionality. As is well-known, the selfdiffusion coefficient exists and well-defined in three dimension for interacting fluids, but it diverges in two dimension. ${ }^{14,15}$ The situation is interesting and complicated, even controversial in 1D where it should have been the simplest. The claim of the existence of a well-defined short time diffusion coefficient in 1D, an expression of which has been given by Lebowitz and Percus ${ }^{11}$ for hard rods and a table which has been given for Lennard-Jones fluids by Bishop et al. ${ }^{13}$ is surprising given that the tagged particle motion is expected to be highly correlated with its nearest neighbors which shall form unsurmountable cages to its motion. Thus the diffusion should show a sub-diffusive behavior in the long time. It has been predicted (and confirmed in simulations). There is, however, an additional aspect to diffusion in 1D which is the effect of noise on diffusion. This later development is motivated by diffusion in zeolites and nanotubes. The diffusion scenario changes drastically in the presence of noise. The interplay between noise and inter-particle interaction has been a subject of several studies. ${ }^{2}$

The Jepsen expression ${ }^{6}$ for the velocity autocorrelation function (VACF) for pure hard rods showed that the long time VACF decays as $1 / t^{3}$, in contrast to the $1 / t^{\mathrm{D} / 2}$ dependence reported for the two $(D=2)$ and three $(D=3)$ dimensions. It is important to note that although the Jepsen solution is exact, it is obtained in the limit of $N \rightarrow \infty$ while keeping the time fixed, where $N$ is the number of the particles present in 
the system. As shown earlier by Hahn and Kärger, ${ }^{2}$ the mean-square displacement (MSD) of a tagged hard rod obeys the following interesting limit: ${ }^{2}$

$$
\left\langle x^{2}\right\rangle_{\infty}=\frac{1}{6}(1-\rho l)^{2} \frac{N}{\rho^{2}},
$$

where $\left\langle x^{2}\right\rangle_{\infty}$ denotes the limiting MSD as $t \rightarrow \infty . l$ is the length of the hard rod and $\rho$ is the number density. Thus, MSD becomes independent of time in the long time. Therefore, the long time diffusion is zero at any density. Numerical calculations show that MSD varies linearly with time till the above saturation occurs. Thus, because $\Delta x$ for any particle is bounded by $N / \rho$, one can indeed talk of a short time diffusion during which the probability distribution of a tagged particle position spreads as a Gaussian function, as occurs in a truly diffusive process. This was discussed by Jepsen himself. However, what has not been realized in earlier studies is that the time dependence of the velocity correlation function (and also of MSD), as given by Jepsen, is valid only to the short time part which depends on the size of the system. Thus, Jepsen solution fails to describe the rich long time decay behavior of $C_{v}(t)$ discussed later in this paper.

Lebowitz and Percus ${ }^{11}$ made an exponential approximation for short time VACF for hard rods and predicted that the short time diffusion should vary as $\rho /(1-\rho l)$, where $\rho$ is the number density of rods of length $l{ }^{10}$ The Jepsen expression also gives a nonzero value of $D .^{6,10,11}$ The values given by Bishop et al. ${ }^{13}$ agree well with the expression of Lebowitz and Percus. ${ }^{11}$

Unlike for hard rods, no analytical solution exists for 1D LJ rods. Earlier, we carried out the MD simulation ${ }^{16}$ of the 1D LJ system to verify the short time results of Bishop et al. ${ }^{3}$ and also to understand the anomalous $1 / t^{3}$ long time behavior of VACF. ${ }^{6}$ In the work reported in Refs. 13 and 16, the upper limit of the integral was selected to be the region where VACF becomes mostly noise. However, in this work we show that the oscillations in VACF persists for a very long time in 1D. By long time we mean two to three orders of magnitude larger than that considered by Bishop et al. Thus the diffusion reported by Bishop et al. can be considered as the short time diffusion. We have also carried out a mode coupling theory analysis to supplement computational efforts on diffusion under a continuous potential.

In addition to the 1D Lennard-Jones system, extensive molecular dynamic simulations of 1D hard rods and 1D repulsive soft spheres are carried out. It is found that at high density, the results are similar to the ones observed in the LJ system. We have also carried out a mode coupling theory analysis in order to understand the rich dynamical behavior of the velocity correlation function. The mode coupling theory has been developed because no exact solution exists for continuous potentials.

Another motivation of the present work is to understand the recent experimental results of Wei et al., ${ }^{1}$ who observed that the particle self-diffusion is non-Fickian for the long periods of time in the single-file diffusion of colloids in onedimensional channels. They have also observed that although the particles execute sizable spatial movements as a function of time, the net mean-square displacement remains nearly zero. This has been attributed to the mutual excluded volume interaction.

The organization of the rest of the paper is as follows. In the next section we describe the simulation details. In Sec. III the simulation results and discussion part is presented. Section IV describes the effects of background noise on the dynamical correlations. Mode coupling theory analysis is presented in Sec. V. We close the paper with a few conclusions in Sec. VI.

\section{SIMULATION DETAILS}

Simulation details remain almost the same as described in our previous study, ${ }^{16}$ except the striking difference that the present simulations are carried out for a very long time. Nevertheless, we mention the simulation method in brief.

The simulation system consists of 1000 Lennard-Jones (LJ) rods placed in a row in one dimension with the initial velocities sampled from the Maxwellian velocity distribution. The rods move according to Newtonian dynamics and interact through the pairwise additive Lennard-Jones potential,

$$
V_{i j}(x)=4 \epsilon\left[\left(\frac{l}{x}\right)^{12}-\left(\frac{l}{x}\right)^{6}\right],
$$

where $i$ and $j$ represents two different LJ rods, $l$ being the length of rod and $\epsilon$ is the measure of the interaction. Mass $(m)$ and length $(l)$ of the rods are scaled to unity. The reduced units being used in simulations for distance, time, temperature, and the density are $x^{*}=x / l, \quad \tau=\sqrt{(m / \epsilon)} l, T^{*}$ $=k_{B} T / \epsilon$, and $\rho^{*}=\rho l$, respectively. The time dependent diffusion $D(t)$, described later, is scaled by $l^{2} / \tau$.

We have studied a wide range of densities starting from $\rho^{*}=0.1$ to $\rho^{*}=0.9$ at $T^{*}=1.0$. This system behaves exactly like the point atoms placed at a distance of $1 / \rho$ interacting in a volume of $V=L-N l$, where $L$ is the length of the simulation box and $N$, the number of LJ rods. Each time the velocities are updated by using Verlet algorithm with $\Delta t$ $=0.0005 \tau$ at all the densities except for $\rho^{*}=0.1$, where we have used $\Delta t=0.002 \tau$.

\section{SIMULATION RESULTS AND DISCUSSION}

Figure 1 shows the MSD as a function of reduced time at $\rho^{*}=0.85$ and $T^{*}=1.0$; inset shows that for a short time. It is important to note that even this short time is 5-10 times larger than the total simulation time of Bishop et al. This figure reveals many interesting results. MSD is linear in time even up to $10 \tau$. Beyond $10 \tau$, the growth in MSD progressively decreases and ultimately starts oscillating around $0.45 l$ which is within $10 \%$ of the value predicted by Eq. (3). This essentially suggests the trapping of rods by their nearest neighbors in $1 \mathrm{D}$. The study of very long time $V A C F$ is also revealed by the same conclusion. In Fig. 2 the VACF is plotted against the reduced time at the same temperature and density as in Fig. 1. While the main figure shows the full decay of VACF, the short time decay of VACF is shown in the inset. Note the small, negative tail which persists for an 


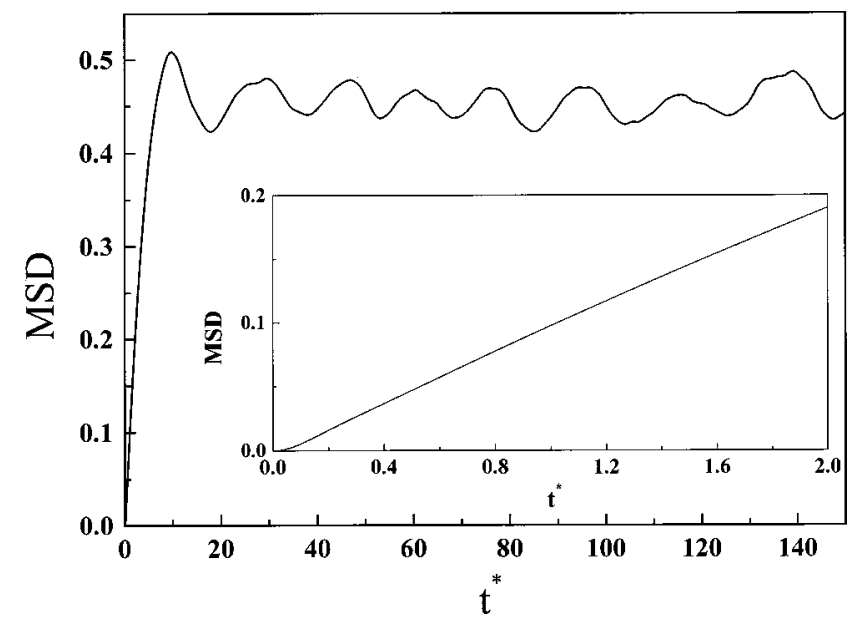

FIG. 1. The mean-square displacement (MSD) obtained from MD simulation is plotted against the reduced time. Inset shows the same for a relatively short time. The reduced temperature and density are 1.0 and 0.85 , respectively.

amazingly long time and is responsible for the zero value of the long time diffusion coefficient. This aspect was missed in earlier simulations.

In order to understand the above aspect, we have carried out a careful analysis of individual trajectories. Figure 3 shows such trajectories of 10 adjacent rods up to very long time, $1000 \tau$ for $\rho^{*}=0.85$ and $T^{*}=1.0$. Curves represent the trajectory of various (adjacent) rods. As can be seen from this figure the motion of the adjacent rods are highly correlated. Such highly cooperative behavior has also been observed recently by Wei et al. ${ }^{1}$ in their experimental study of single file diffusion of colloids in a one-dimensional system. They have observed the trajectories of eight neighboring particles in a channel. The trajectories plotted in Fig. 3 look quite similar to those reported in the experimental study of Wei et al. More importantly, all the adjacent particles are found to move together, giving rise to transient longitudinal flow or current. These flows cause quite large displacement, but positive flows are as likely as negative flows, so that the mean displacement remains the same. The large flows are

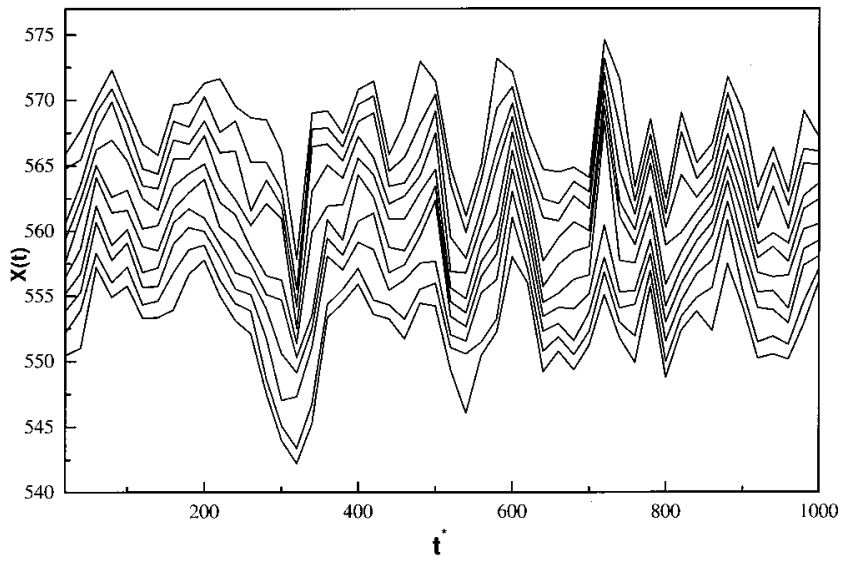

FIG. 3. Typical trajectories of 10 adjacent rods obtained from a very long MD simulations of $1 \mathrm{D} \mathrm{LJ}$ rods. $T^{*}=1.0$ and $\rho^{*}=0.85$. Note the highly correlated motion of the adjacent particles, which follows from the transient longitudinal current modes.

intermittent; that is, they appear only after large intervals. There are smaller flows during this interval.

In order to understand the earlier theoretical prediction of a finite, nonzero short time diffusion constant, we have plotted the time dependent diffusion constant against reduced time in Fig. 4. In this case the reduced temperature and densities are also 1.0 and 0.85 , respectively. The time dependent diffusion coefficient can be defined by any of the following two expressions:

$$
D(t)=\frac{1}{2} \frac{d}{d t}\left\langle\Delta x^{2}(t)\right\rangle
$$

and

$$
D(t)=\int_{0}^{t} d \tau\langle v(0) v(\tau)\rangle
$$

where $\left\langle\Delta x^{2}(t)\right\rangle$ is the mean-square displacement of a tagged particle, $v(\tau)$ is the velocity at time $\tau$.

Both the definitions give essentially the same result; Fig. 4 has been produced by using the velocity correlation function [i.e., by using Eq. (6)]. Note the slow approach of $D(t)$

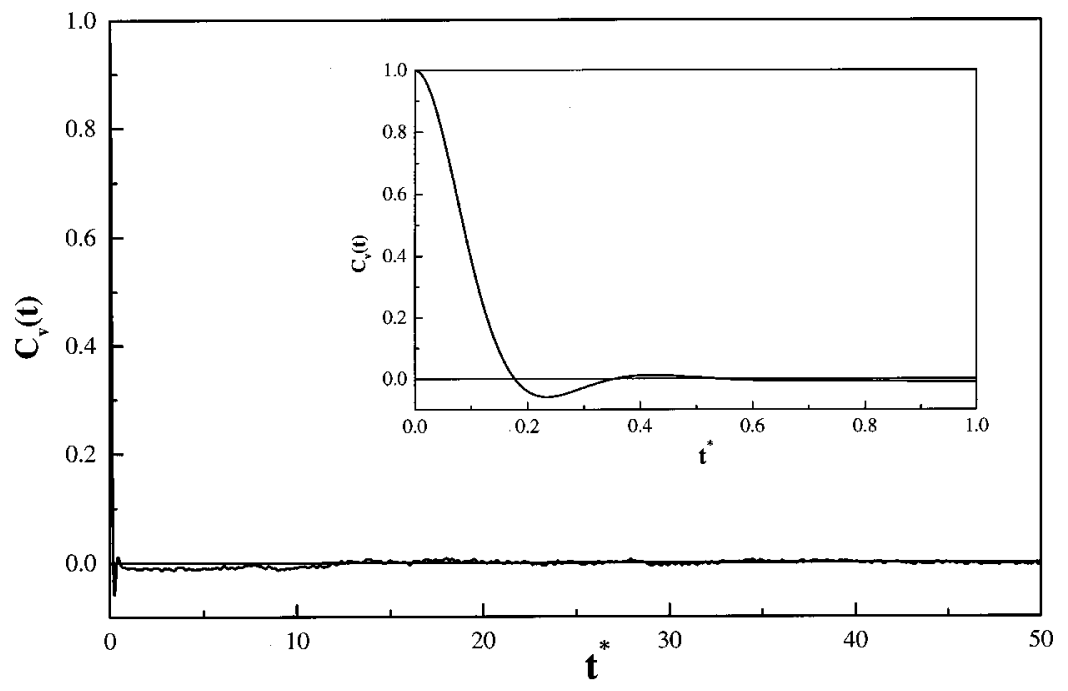

FIG. 2. Normalized VACF obtained from simulations is plotted against the reduced time at reduced density 0.85 and $T^{*}=1.0$. Main figure shows the full decay of VACF while the short time decay is shown in the inset. 


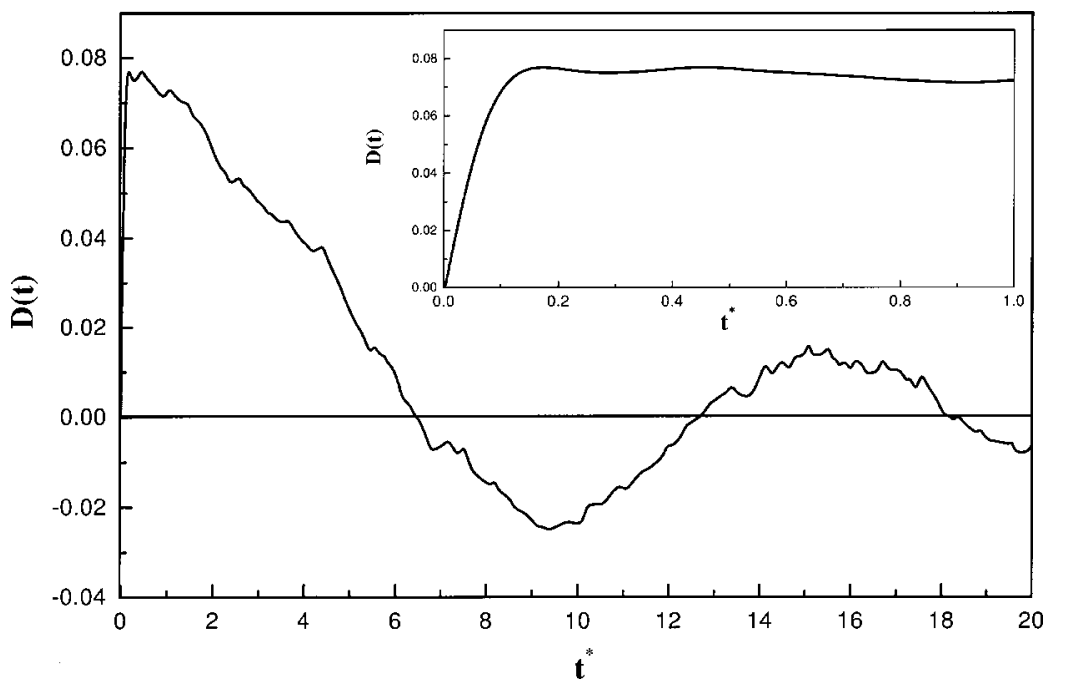

FIG. 4. The time dependent diffusion coefficient $D(t)$ obtained from the simulated VACF [by using Eq. (6)] is shown against the reduced time. Main figure shows the $D(t)$ for the long time, while the short time $D(t)$ is shown in the inset. This figure clearly shows that in the long time, diffusion goes to zero. $T^{*}=1.0$ and $\rho^{*}=0.85$. to zero. Earlier simulations have considered only the short time region where $D(t)$ shows a plateau (shown in the inset of Fig. 4), and seem to have missed the long time behavior (main figure).

We have also carried out extensive molecular dynamic simulations with 1D hard rod and 1D repulsive soft sphere (repulsive) potentials; in the latter case the interaction potential has $(l / x)^{12}$ form. In both these cases, the quantitative features change somewhat, but qualitative aspects remain the same: (a) $D(t)$ goes to zero in the long time; (b) a short time diffusion coefficient can be defined; and (c) VACF exhibits a small negative region for a long time. We further noticed that at high density, Lennard-Jones rods behave like hard rods. However, at intermediate densities (for, e.g., at $\rho^{*}=0.6$ ) the LJ system does not show any negative region in VACF whereas the hard rods show a negative region. This can be attributed to the pronounced backscattering in the case of hard rods.

The cross-over of MSD from the linear short time behavior to the oscillatory, time independent long time behavior is of considerable interest. Our simulations show that the cross-over is strongly density dependent. It gets shifted to longer and longer times as the density is increased. In addition, the cross-over also shows strong system size dependence. The latter has already been discussed by Hahn and Kärger, ${ }^{2}$ who showed that the cross-over MSD scales with the number of particles $(N)$ as given by Eq. (3), which seems to describe the cross-over reasonably well. Thus in the limit of large $N$, the cross-over would occur at a very large value of MSD. Thus, for an infinite system, the short time diffusion can indeed become a relevant quantity. However, the diffusion coefficient itself is zero in the strict sense because of definition in Eq. (1). Thus, one should discuss diffusion only in terms of a time dependent diffusion, $D(t)$. If the short time diffusion, $D_{\text {short }}$ is known, then one can approximately find the cross-over time from Eq. (3) and it is given by

$$
t_{\text {cross }} \approx \frac{N}{12 D_{\text {short }}}\left(\frac{1}{\rho^{*}}-1\right)^{2} .
$$

Thus, MSD shows two different time regimes.
Jepsen's analytical solution predicts an $N$-independent finite diffusion coefficient. As this solution was obtained in the thermodynamic limit, it is clear that this result corresponds to the limit of $N \rightarrow \infty$. This point is also clear from the work of Lebowitz and Percus. ${ }^{11}$ Thus, it is rather important to explore the effects of finite $N$, both on the diffusion and the velocity correlation function, in one-dimensional interacting systems. This will allow an in-depth of understanding of the approach to the thermodynamic limit. Note that the slow approach to the thermodynamic limit is a hallmark of the one-dimensional systems.

In Fig. 5 we show the system size dependence of the velocity time correlation function at density $\rho^{*}=0.6$. We have not discussed the system size dependence of MSD as it has been discussed earlier by Hahn and Kärger. The simulated VACF shown in Fig. 5 shows some remarkable features. For a smaller system the negative dip at intermediate time is quite pronounced while the long time tail is nearly absent. As the size of the system is increased, the negative dip becomes smaller but a very long time, persisting tail appears. The time dependence of this long time tail is weaker than the well-known $t^{-3}$ tail often discussed in the literature. Thus the reason for the vanishing long time diffusion coefficient is rather different for the small and large systems. In smaller systems the back flow is stronger and sets in earlier and then decays quickly. In larger systems the back flow is weaker but it persists for a longer time. This is also clearly manifested in the system size dependence of the MSD. ${ }^{2}$

\section{EFFECTS OF BACKGROUND NOISE}

Diffusion in the single file system is often described in theoretical studies in terms of random walk of an isolated particle which is under the influence of a uniform background noise. In this case, MSD is known to follow a relation such as ${ }^{8}$

$$
\left\langle x^{2}(t)\right\rangle=2 F \sqrt{t}
$$

where $F$ is the mobility factor of the single file diffusion. Inter-particle interactions modify the ideal behavior. ${ }^{8}$ It has 


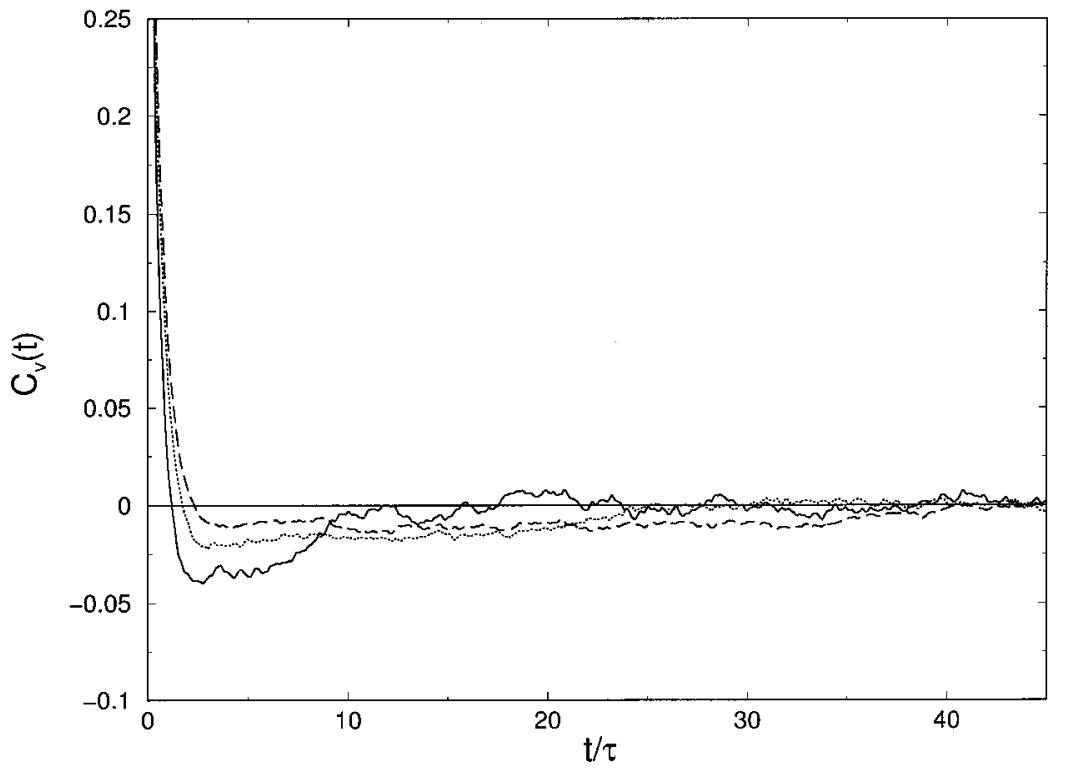

FIG. 5. The simulated velocity time auto correlation function for different system size at $\rho^{*}=0.6$ and $T^{*}$ $=1.0$ is plotted against reduced time. The solid, short dashed and the long dashed lines corresponds to VACF obtained for $N=32,64$, and 128 , respectively. been suggested that the MSD of a particle in the single file system with interactions can be related to the absolute value $\langle|s|\rangle$ for the shift of a noninteracting particles present in the same one-dimensional channel by, 2,9

$$
\left\langle x^{2}\right\rangle=\left(\frac{1-\rho^{*}}{\rho^{*}}\right)\langle|s|\rangle .
$$

Note that the above considerations apply where the particles in single file are not only interacting among themselves, but are also subject to random noise.

The experiments of Wei et al. ${ }^{1}$ fall in the intermediate domain where both interparticle interactions and the background noise are important. Thus, the long time diffusion is certainly zero for this experimental system. What is not clear is the existence of the time ranges where Eqs. (3) and (7) could be valid. One still expects a time range where a short time diffusion exists which goes over to a $\sqrt{t}$ type MSD behavior at the intermediate time.
Levitt $^{8}$ and Percus ${ }^{9}$ have earlier made the remarkable prediction that in a one-dimensional system with a random background noise, in the long time limit, $p(x, t)$ (the probability of finding a particle at $x$ at time $t$ that was at $x=0$ at $t=0)$, is proportional to $t^{-1 / 4} \exp \left(-x^{2} /\left\langle x^{2}(t)\right\rangle\right)$, where $\left\langle x^{2}(t)\right\rangle \rightarrow \gamma t^{1 / 2} / \rho$. Therefore, the MSD is predicted to have a $\sqrt{t}$ dependence. Hahn and Kärger ${ }^{2}$ incorporated the effect of random background noise by randomly changing the particle velocity. With this randomized velocity they found that, although in the intermediate time the MSD has a linear dependence on $t$, there is a crossover to $\sqrt{t}$ dependence in the long time. Thus the background noise has a strong effect on the behavior of MSD (and also VACF) and thus on the dynamics of the system. However, the effects on the VACF have not been investigated.

In order to understand this point we have carried out further MD simulation where the effect of random noise is taken into account by randomly changing the velocity after

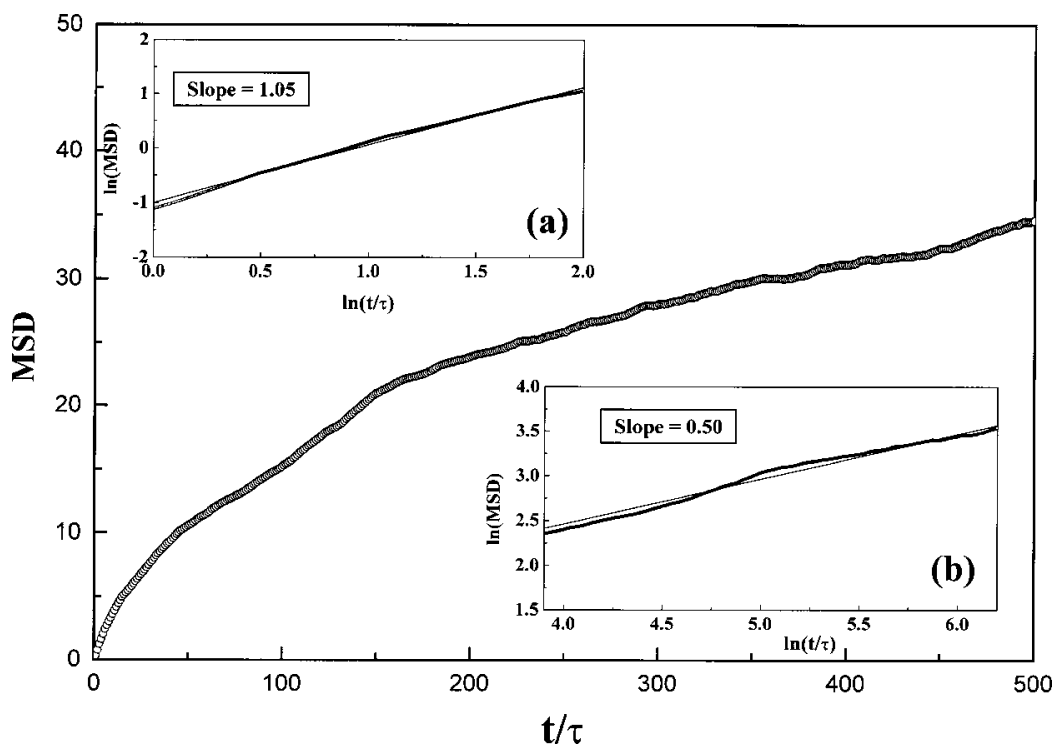

FIG. 6. The time dependence of the mean-square displacement (MSD) obtained from MD simulation in presence of the background noise is plotted (main figure). Inset (a) shows that in the intermediate time MSD has a linear dependence on time, while inset (b) shows that in the long time it goes as $\sqrt{t}$. The reduced temperature and density are 1.0 and 0.6 , respectively. 


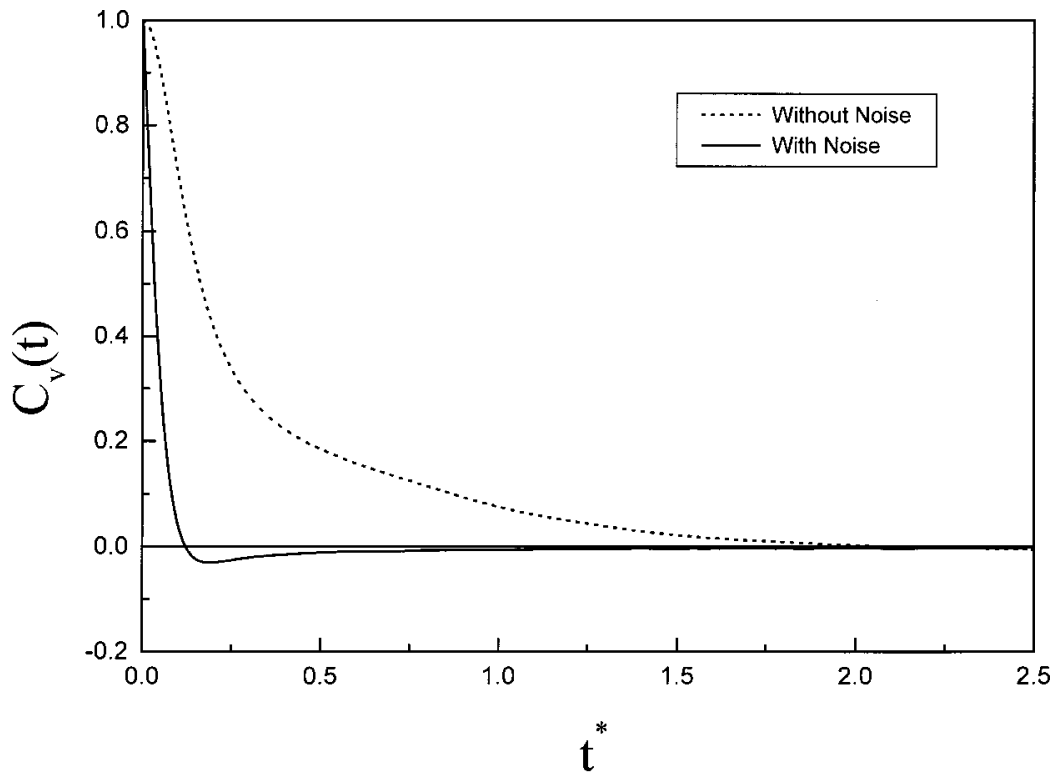

FIG. 7. The normalized velocity autocorrelation function both in presence (full line) and absence (dashed line) of the background noise is plotted as a function of reduced time at $\rho^{*}=0.6$ and $T^{*}=1.0$. regular time intervals, $N$, with $N=100 \tau$ or $1000 \tau$. This was the procedure adopted by Hahn and Kärger. ${ }^{2}$ The time dependence of MSD is shown in Fig. 6. Simulation results show that although, in the intermediate time, the MSD has a linear dependence on time, in the long time it goes as $\sqrt{t}$. This is in agreement with theoretical predictions. However, the new result is the rather sharp cross-over form linear to $\sqrt{t}$ dependence of mean-square displacement.

It is also natural that the behavior of VACF will be affected by this random noise. This has been addressed to by Levitt and Percus in two well-known papers. They predicted that the random noise will give rise to a power-law long time decay of the VACF, $C_{v}(t) \propto t^{-\alpha}$, with $\alpha=3 / 2 .{ }^{8,9}$ However, we are not aware of any detailed computational study of the effects of random noise on VACF. Figure 7 displays the decay behavior of this correlation both in the presence and in the absence of the random noise. As can be seen from this figure, the decay of random noise modulated correlation function is much faster. This behavior is of course expected in the presence of the random noise. What was not expected is the total change in the intermediate time. Note also that the small amplitude long time tail in the VACF disappears completely in the presence of the random noise. Instead a tail appears which can be fitted to a power-law form with an exponent equal to $\alpha=1.3 \pm 0.2$. Nevertheless, in this case one can also define a short time diffusion.

In order to understand the effect of noise on the particle trajectories, we have carried out a careful analysis of individual trajectories, as before. Figure 8 shows such trajectories of 10 adjacent rods up to very long time, $700 \tau$ for $\rho^{*}=0.85$ and $T^{*}=1.0$. If one study a one-dimensional system in the presence of noise, one finds that the noise dampens oscillations in the trajectories as shown in Fig. 8 (compare with Fig. 3). Although the nature of the oscillations observed here is in agreement with the recent experimental study of Wei et al., ${ }^{1}$ one needs to do further detailed investigation in this direction.

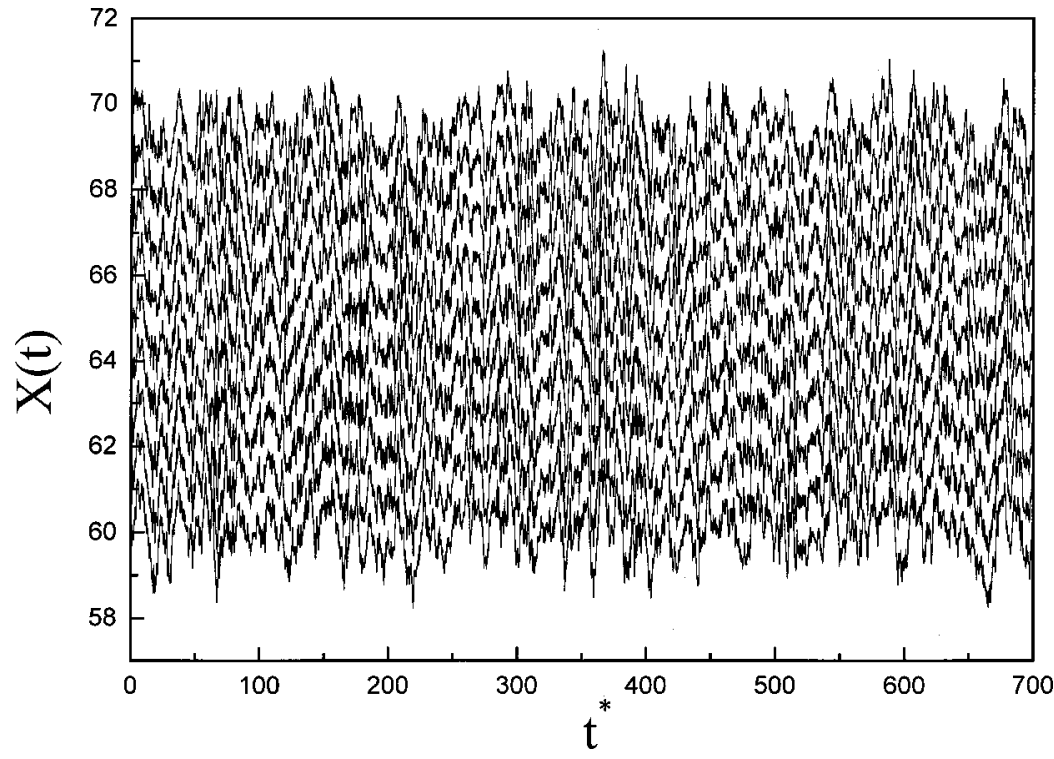

FIG. 8. Typical trajectories of 10 adjacent rods in presence of background noise obtained from a very long MD simulations of $1 \mathrm{D} \mathrm{LJ}$ rods are shown. $T^{*}=1.0$ and $\rho^{*}=0.85$. Note that in presence of noise, the correlated motion of the adjacent particles lost. 


\section{MODE COUPLING THEORY ANALYSIS}

The success of the mode coupling theory in describing the diffusion both in 3D and 2D motivated us to extend it to the $1 \mathrm{D}$ systems. MCT correctly predicts the diffusion coefficient in 3D and could predict that in $2 \mathrm{D}$, although the time dependent diffusion exists, the long time diffusion coefficient diverges due to the transverse current mode.

As observed in simulations (presented in the previous section), in 1D the VACF shows a long time negative tail and the time dependent diffusion in the long time is oscillatory. The total diffusion coefficient is found to be zero. It is clearly nontrivial to capture this behavior from a microscopic theory and mode coupling theory is the only theoretical scheme available to analyze these results. In order to calculate either VACF or the diffusion coefficient with MCT, we need the two particle direct correlation function, $c(x)$, and the radial distribution function, $g(x)$. Here $x$ denotes the separation between the centers of two LJ rods. In order to make the calculations robust we have used the $g(x)$ obtained from simulations. The frequency $(z)$ dependent velocity correlation function $C_{v}(z)$ is related to the frequency dependent friction by the following generalized Einstein relation, ${ }^{17}$

$$
C_{v}(z)=\frac{k_{B} T}{m(z+\zeta(z))},
$$

where $\zeta(z)$ is the frequency dependent friction.

In mode coupling theory, the full friction is decomposed into a short and a long time part. Short time part arises from the binary collisions of tagged particle with the surrounding solvents and the long time part originates from the correlated recollisions. Final expression for the frequency dependent friction used to calculate both VACF and time dependent diffusion is given by ${ }^{18}$

$$
\zeta(z)=\zeta^{B}(z)+\zeta^{R}(z)
$$

where $\zeta^{B}(z)$ is the binary part of the zero frequency friction, $\zeta^{R}(z)$ is the ring collision term, which contains the contributions from the repeated collisions to the total friction. The expression of $\zeta^{R}(z)$ in 1D will be similar to that in 3D and $2 \mathrm{D}^{16,17}$ but without the presence of the term that contains the contribution from the transverse current to the total friction. Replacing the expression of $\zeta^{R}(z)$ in the above equation the total friction can be written as

$$
\zeta(z)=\frac{\zeta^{B}(z)+R_{\rho \rho}(z)-\zeta^{B}(z) R_{\rho l}(z)}{1+R_{\rho l}(z)+\zeta^{B}(z) R_{L}(z)} .
$$

In the above expression $R_{\rho \rho}(z)$ contains the coupling to the density and is given by

$$
\begin{aligned}
R_{\rho \rho}(t)= & \frac{\rho k_{B} T}{m} \int\left[d q^{\prime} /(2 \pi)\right] q^{\prime 2}\left[c\left(q^{\prime}\right)\right]^{2} \\
& \times\left[F^{s}\left(q^{\prime}, t\right)-F_{0}^{s}\left(q^{\prime}, t\right)\right] F\left(q^{\prime}, t\right) .
\end{aligned}
$$

$c(q)$ is the Fourier transform of $c(x) . R_{l l}(z)$ contains the contribution of the longitudinal current while $R_{\rho l}$ includes the simultaneous coupling to the density and longitudinal current modes. These can be expressed by the following expressions in one dimension (by following the similar procedure used in $3 \mathrm{D}),{ }^{16,17}$

$$
\begin{aligned}
R_{l l}(t)= & \frac{1}{\rho} \int\left[d q^{\prime} /(2 \pi)\right]\left[\gamma_{d}^{l}\left(q^{\prime}\right)\right. \\
& \left.+\left(\rho q^{2} / m \beta\right) c\left(q^{\prime}\right)\right]^{2} \omega_{0}^{-4} \\
& \times\left[F^{s}\left(q^{\prime}, t\right)-F^{0}\left(q^{\prime}, t\right)\right] C_{l}\left(q^{\prime}, t\right),
\end{aligned}
$$

and

$$
\begin{aligned}
R_{\rho l}(t)= & \int\left[d q^{\prime} /(2 \pi)\right] c\left(q^{\prime}\right)\left[\gamma_{d}^{l}(q)\right. \\
& \left.+\left(\rho q^{2} / m \beta\right) c\left(q^{\prime}\right)\right] \omega_{0}^{-2} \\
& \times\left[F^{s}\left(q^{\prime}, t\right)-F^{0}\left(q^{\prime}, t\right)\right] \frac{d}{d t} F\left(q^{\prime}, t\right),
\end{aligned}
$$

where $\gamma_{d}^{l}(q)$ is the distinct part of the second moment of the longitudinal current correlation function, which is given by the following equation:

$$
\gamma_{d}^{l}(q)=-\frac{\rho}{m} \int d x \cos (q x) g(x) \frac{d^{2}}{d x^{2}} v(x),
$$

and $\omega_{0}$ is the well-known Einstein frequency in 1D and is given by

$$
\omega_{0}^{2}=\frac{\rho}{m} \int d x g(x) \frac{d^{2}}{d x^{2}} v(x) .
$$

$\zeta^{B}(t)$ is the binary part of the friction whose expression is given by

$$
\zeta^{B}(t)=\omega_{0}^{2} \exp \left(-t^{2} / \tau_{\zeta}^{2}\right) .
$$

The relaxation time $\tau_{\zeta}$ is determined from the second derivative of $\zeta^{B}(t)$, which is given by the following equation:

$$
\begin{aligned}
\frac{\omega_{0}^{2}}{\tau_{\zeta}^{2}}= & \frac{\rho}{3 m^{2}} \int d x \frac{d^{2}}{d x^{2}} v(x) g(x) \frac{d^{2}}{d x^{2}} v(x) \\
& +\frac{1}{4 \pi \rho} \int d q \gamma_{d}^{l}(q)(S(q)-1) \gamma_{d}^{1}(q),
\end{aligned}
$$

the static structure factor, $S(q)$, appearing in the above expression is calculated by using the one-dimensional Fourier transform of the radial distribution function.

The longitudinal current correlation function, $C_{l}(q, t)$, is related to the dynamic structure factor by the following expression:

$$
C_{l}(q, t)=-\frac{m^{2}}{q^{2}} \frac{d^{2}}{d t^{2}} F(q, t) .
$$

It is important to note that at sufficiently long times the only significant contribution to the integral of Eq. (14) arises from small wave numbers. Note that the only existing current modes are the longitudinal ones and are directly related to $F(q, t)$ through the above equation.

As mentioned earlier, we present a MCT analysis of the rich time dependence of the $C_{v}(t)$ as observed in the simulations. A MCT analysis of the $t^{-3}$ decay of the VACF was presented earlier. ${ }^{16}$ Although the analysis was not complete, it showed that the origin of the $t^{-3}$ decay was the $R_{l l}$ term. In reality the situation is more subtle with all the terms hav- 


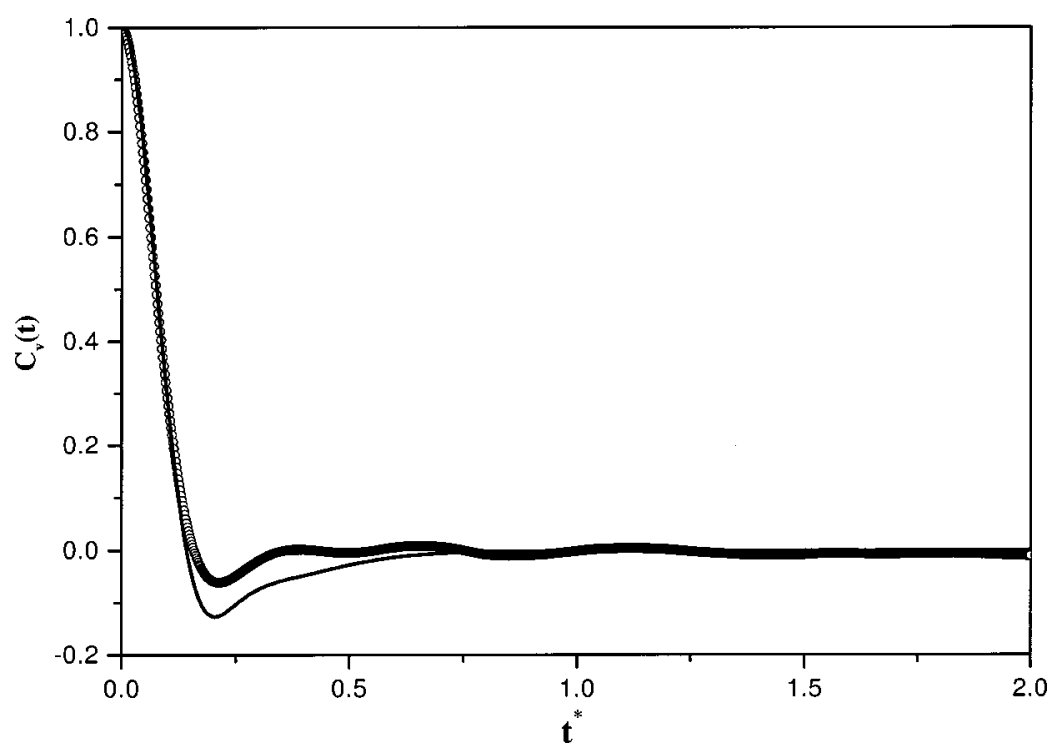

FIG. 9. The normalized velocity autocorrelation function obtained from the fully self-consistent mode coupling theory (line) is plotted against the reduced time at $\rho^{*}=0.85$ and $T^{*}=1.0$. For comparison the result of simulation (symbols) is also shown.

ing different time scale of decay. Also note that a factor of $q^{2}$ was missing in Eq. (19) of Ref. 16, although the final form had the correct time dependence. The presence of $q^{2}$ will lead to a different power of $t$.

To understand the origin of the complex time dependence of VACF, we have analyzed all the terms in $\zeta^{R}(z)$ in their asymptotic limit. In all of these analysis the most important assumption is a Gaussian ansatz for $F(q, t)$. The simulated $F(q, t)$ is found to decay mostly as a Gaussian function, given by

$$
F(q, t) \approx \exp \left(-a q^{2} t^{2}\right) .
$$

The other assumptions are the following. Since in sufficiently long time the significant contribution arises from small and intermediate wave numbers, the wave number dependent vertex functions in the integrals [Eq. (19)] are replaced by constant values which they attain at $q \rightarrow 0$ limit. The decay of $F_{s}(q, t)$ is assumed to be slow and it is replaced by 1 . With the abovementioned assumptions the expression for $R_{\rho \rho}(t), R_{l l}(t)$, and $R_{\rho l}(t)$ can be written as

$$
\begin{aligned}
R_{\rho \rho}(t)= & C_{1} \int d q q^{2} \exp \left(-q^{2} t^{2}\right) \\
R_{l l}(t)= & C_{2} \int d q C_{1}(q, t)+C_{3} \int d q q^{2} C_{l}(q, t) \\
& +C_{4} \int d q q^{4} C_{l}(q, t),
\end{aligned}
$$

and

$$
R_{\rho l}(t)=C_{5} \int d q \frac{d}{d t} F(q, t)+C_{6} \int d q q^{2} \frac{d}{d t} F(q, t) .
$$

In the above equations $C_{i}(i=1,2 \ldots . ., 6)$ are constants which are independent of $q$ and $t$. Now we substitute Eq. (21) in Eq. (22) and perform the wave number integration to get

$$
R_{\rho \rho}(t)=\frac{C_{1} \sqrt{\pi}}{4} t^{-3}
$$

Similarly we substitute Eq. (21) in Eq. (20) and then substitute the expression of $C_{l}(t)$ in Eq. (23). After performing the wave number integration, Eq. (23) can be written as

$$
R_{l l}(t)=-C_{3} m \sqrt{\pi} t^{-3}-3 C_{4} m \sqrt{\pi} t^{-5},
$$

where the first term on the right hand side does not survive.

If we replace Eq. (21) in Eq. (24) and perform the wave number integration we get

$$
R_{\rho l}(t)=-\frac{C_{5} \sqrt{\pi}}{2} t^{-2}-\frac{3 C_{6} \sqrt{\pi}}{4} t^{-4} .
$$

Note that in the above equations the pre-factors come with both positive and negative signs. The equations in the time plane need to be Laplace inverted to get the frequency dependent full friction [given by Eq. (12)]. A Laplace transform of Eq. (10) with the full $\zeta(z)$ will give the VACF. Thus it is evident that the time dependence of $R_{\rho \rho}, R_{l l}$, and $R_{\rho l}$ will not directly get translated to the VACF and from the above expressions it is not possible to make an estimate of the weightage of the different terms. But it can be argued that due to complex time dependence of the recollision term the time dependence of $C_{V}(t)$ will not be simple and it will have multiple time scales of decay, in agreement with the simulation studies.

While the above analysis could explain the asymptotic behavior of the VACF, the decay over the entire time range can be explained only by a fully self-consistent mode coupling theory calculation. In the following we present the results obtained from such a self-consistent calculation. The only approximation made in these calculations is that the $q$ dependence of $\Gamma(q)$ can be neglected. While this approximation makes the agreement imperfect, one can still see the basic behavior to emerge from the MCT analysis.

In Fig. 9 the normalized VACF obtained from the fully self-consistent mode coupling theory at $\rho^{*}=0.85$ and $T^{*}$ $=1.0$ is plotted against the reduced time. For comparison, the VACF obtained from simulation is also shown in the same figure. As can be seen from the figure, similar to the 


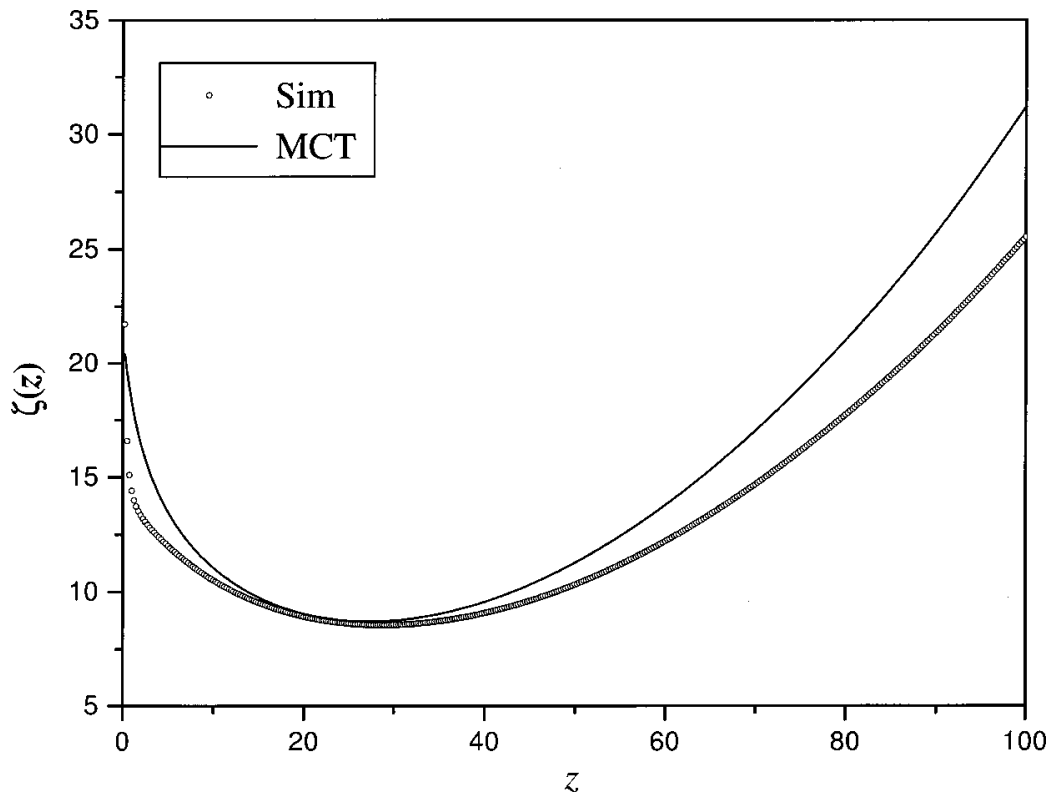

FIG. 10. The frequency dependent friction obtained from the fully self-consistent mode coupling theory (line) along with the same obtained from simulation (symbols) is plotted at $\rho^{*}=0.85$ and $T^{*}=1.0$. simulation result (symbols) MCT (line) also shows a pronounced negative VACF at short to intermediate times. To understand both the short and the long time decay of VACF in more detail, the frequency dependent friction (obtained from both simulation and MCT) at the same density and temperature is plotted in Fig. 10 as a function of frequency (z). As can be seen from this figure the MCT prediction (line) is reasonably in good agreement with that obtained from the simulation (symbols).

However, MCT fails to provide the approach to the zero value of the long time diffusion coefficient, at finite $N$. The reason is the same as in Jepsen-the thermodynamic limit has been invoked in the calculation of $C_{v}(t)$. In the ideal scenario, one should calculate a $C_{v}(t)$ that depends on $N$ and $L$ and one should take the limit $N \rightarrow \infty, L \rightarrow \infty$ with $\rho=N / L$ = fixed later.

Note that for applications, the above is not a deficiency since one is interested in the short time diffusion which is the meaningful quantity in the thermodynamic limit.

MCT is known to give reliable results in the thermodynamic limit, particularly in normal liquids (that is, away from the glass transition temperature). In the MCT the system size dependence is usually studied by introducing a cutoff in the lower limit of the wave vector $k$ in the mode coupling integrals. This gives a satisfactory explanation of the $N$ dependence of diffusion in $2 \mathrm{D} .{ }^{15}$ In the present case, such an approach will obviously provide only a weak $N$ dependence, because here the contribution comes primarily from the intermediate $k$. An important source of the $N$ dependence in 1D systems (unlike that in 2D and 3D systems) could be the strong system size dependence of the the radial distribution function and also the two particle direct correlation function.

\section{CONCLUSIONS}

In conclusion, we have shown that in a one-dimensional LJ system diffusion indeed goes to zero in the limit of very long time (which, of course, needs to be defined properly), in contrast to earlier studies ${ }^{13}$ that report finite nonzero diffu- sion in 1D by performing only short time MD simulations. Our conclusions are in agreement with studies of Hahn and Kärger, ${ }^{2}$ except that we have presented the analysis of the velocity time correlation function and a more detailed description of MSD. We find that MSD shows an oscillatory time dependence which is because of the trapping of the rods by their nearest neighbors in 1D at longer times, which is also consistent with the recent experimental results. ${ }^{1}$ In addition, we have presented a fully self-consistent mode coupling theory which captures much of the rich dynamical behavior of the velocity correlation function and also of the frequency dependent friction in one-dimensional strongly interacting systems.

An important outcome of the present study is the discovery of the very slow long time decay of the velocity correlation function. It is this negative ultra-slow decay which eventually leads to the vanishing of the diffusion coefficient. The slow decay observed here is much slower than the $t^{-3}$ decay often discussed earlier. The slow decay becomes progressively less in amplitude and longer in time span as the size of the system is increased. In addition, the first time $t_{\text {fneg }}$ that $C_{v}(t)$ becomes negative moves to longer time as $N$ is increased. It is likely that there exists a scaling relation between $t_{\text {fneg }}$ and $N$, but this requires further study. Unfortunately, the Jepsen solution is not very useful for this purpose because of the thermodynamic limit already employed. The present simulations suggest that the amplitude of the first negative dip scales with $N$ as $N^{-\nu}$ where $\nu \approx 1.0$. We know from simulations that the short time diffusion is independent of $N$. Thus, the integral of the long time negative tail must also be independent of $N$, because the diffusion must be zero in the limit of $t \rightarrow \infty$, at fixed $N$. Since the pre-factor of the negative tail decreases as $N^{-\nu}$, the contribution from the rest of the integrand must increase as $N^{\nu}$. Therefore, the tail of VACF must persist up to a time that grows with $N$, while the amplitude of the tail itself decreases as $N^{-\nu}$. The situation is even more interesting in MSD. Here a lengthening of the decay of $C_{v}(t)$ implies a corresponding lengthening of the 
cross-over region from the linear short time behavior to the flat long time behavior. This is indeed seen in simulation.

The above provides a physical picture behind the approach of the system dynamics to the large $N$ limit. Thus, the theories that invoke a $N \rightarrow \infty$ limit obtain only the short time diffusion. However, it is a bit surprising that such rich dynamics at small $N$ as observed here are totally missed if the thermodynamic limit is taken!

This $N$ dependence of the velocity time correlation function is intimately connected with the validity of the GreenKubo relation. We find no signature of the breakdown of this relation here - the diffusion obtained from MSD agrees with that obtained from the time integral of the velocity correlation function, for finite $N$ simulated here.

The present study left several questions unanswered. We have not yet found a way to incorporate the effects of random noise in the mode coupling theory calculation of $C_{v}(t)$. The $N$ dependence of the tail of the $C_{v}(t)$ is also not understood.

\section{ACKNOWLEDGMENTS}

Professor Stuart Rice is acknowledged for drawing attention to the works on single file system (Refs. 1 and 8). The authors thank Professor S. Yashonath for several discus- sions. This work is supported in parts by grants from DST and CSIR, India. G.S. thanks CSIR, for a Research Fellowship.

${ }^{1}$ Q.-H. Wei, C. Bechinger, and P. Leiderer, Science 287, 625 (2000).

${ }^{2}$ K. Hahn and J. Kärger, J. Phys. Chem. 100, 316 (1996); K. Hahn, J. Kärger, and V. Kukla, Phys. Rev. Lett. 76, 2762 (1996).

${ }^{3}$ J. Kärger and D. M. Ruthven, Diffusion in Zeolites and Other Microporous Solids (Wiley, New York, 1992).

${ }^{4}$ N. Y. Chen, T. F. Degnan, and C. M. Smith, Molecular Transport and Reaction in Zeolites (VCH, New York, 1994).

${ }^{5}$ L. V. C. Rees, Proceedings of the 10th International Zeolite Conference (Garmisch-Partenkirchen, 1994).

${ }^{6}$ D. W. Jepsen, J. Math. Phys. 6, 405 (1965).

${ }^{7}$ M. Bishop and B. J. Berne, J. Chem. Phys. 59, 5337 (1973).

${ }^{8}$ D. G. Levitt, Phys. Rev. A 8, 3050 (1973).

${ }^{9}$ J. K. Percus, Phys. Rev. A 9, 557 (1974).

${ }^{10}$ J. W. Haus and H. J. Raveche, J. Chem. Phys. 68, 4969 (1978).

${ }^{11}$ J. L. Lebowitz and J. K. Percus, Phys. Rev. 155, 122 (1967); J. L. Lebowitz, J. K. Percus, and J. Sykes, ibid. 171, 224 (1968).

${ }^{12}$ E. H. Lieb and C. Mattice, Mathematics Problems in One Dimension: Exactly Solvable Models of Interacting Particles (Academic, New York, 1966).

${ }^{13}$ M. Bishop, M. Derosa, and J. Lalli, J. Stat. Phys. 25, 229 (1981).

${ }^{14}$ B. J. Alder and T. E. Weinwright, Phys. Rev. A 1, 18 (1970).

${ }^{15}$ S. Bhattacharyya, G. Srinivas, and B. Bagchi, Phys. Lett. A 266, 394 (2000).

${ }^{16}$ G. Srinivas and B. Bagchi, J. Chem. Phys. 112, 7557 (2000).

${ }^{17}$ B. Bagchi and S. Bhattacharyya, Adv. Chem. Phys. 116, 67 (2001).

${ }^{18}$ L. Sjogren and A. Sjolander, J. Phys. C 12, 4369 (1979). 